convulsions. Neurology 2012 Aug 21;79(8):777-84). (Response and reprints: Dr MattKievit. E-mail: j.a.maat@erasmusmc.nl).

COMMENT. PRRT2 mutations are the major cause of PKD or ICCA, but they are not involved in the etiology of febrile convulsions and migraine. Paroxysmal kinesigenic dyskinesia/choreoathetosis is characterized by brief attacks of involuntary movements (dystonia, chorea, athetosis, and ballism), precipitated by a kinesigenic trigger such as sudden movement or startle. Attacks last $<1$ minute, without loss of consciousness; they begin during childhood, and are controlled by anticonvulsant medication. Neurologic examination and MRI are normal, and ictal EEG shows nonspecific abnormalities. PKD is sporadic or familial. Familial cases have an autosomal dominant transmission with incomplete penetrance. PKD may be associated with BFIC or ICCA and with migraine.

\title{
UTILITY OF THE ELECTROENCEPHALOGRAM
}

\section{EEG IN PREDICTION OF EARLY NEURODEVELOPMENT OF PRETERM INFANTS}

Researchers at Anjo Kosei Hospital and other centers in Japan and at Washington University, St Louis, MO, USA studied the prognostic value of conventional EEG for the identification of preterm infants admitted to the Anjo neonatal ICU and at risk for adverse neurodevelopment. Serial EEG recordings were conducted during 3 time periods, at least once each within days 6 (first period), during days 7 to 19 (second period), and days 20 to 36 (third period). Neurodevelopment outcomes were assessed at a corrected age of 12 to 18 months. Of 333 preterm infants ( $<34$ weeks' gestation), $33(10 \%)$ had developmental delay and $34(10 \%)$ had cerebral palsy. In the infant's 780 EEG records studied between 2002 and 2008, abnormalities were significantly predictive of developmental delay and cerebral palsy at all 3 time periods. Acute stage background EEG abnormalities were graded as mild, moderate, and severe, and were characterized as suppressed with decreased continuity, lower amplitude, and/or attenuated fast-wave. Chronic stage background was characterized by a disorganized pattern with sharp waves or a dysmature pattern. The grade of EEG abnormalities correlated with the incidence of developmental delay or cerebral palsy in all periods $(p<0.001)$. EEG abnormality in the second period was an independent predictor of developmental delay and cerebral palsy. (HayashiKurahashi N, Kidokoro H, Kubota T, et al. EEG for predicting early neurodevelopment in preterm infants: an observational cohort study. Pediatrics 2012 Oct;130(4):e891-7). (Respond: Hiroyuki Kidokoro MD, Department of Pediatrics, Washington University in St Louis, 660 South Euclid Ave, St Louis, MO 63110. E-mail: kidokoro_h@kids.wustl.edu).

COMMENT. EEG abnormalities within the first month of life of preterm infants significantly predict adverse neurodevelopment at age 12 to 18 months. The EEG has prognostic value independent of neuroimaging and clinical risk factors. A disorganized EEG pattern precedes abnormalities on ultrasound and is a better prognostic marker in the neonatal care setting (Kidokoro H et al. Neuropediatrics 2010 Dec;41(6):241-5). 